\title{
New Urban Vertical Axis Wind Turbine Design
}

\author{
F. Frunzulica ${ }^{1,2}$, A. Dumitrache ${ }^{2}$ and A. M. Cismilianu ${ }^{3}$ \\ ${ }^{1}$ Department of Aerospace Engineering \\ POLITEHNICA University of Bucharest \\ Polizu Street no.1-6, RO-011061 Bucharest, Romania \\ Phone/Fax number:+40-722.894.399/+40-21-318.24.33, e-mail: ffrunzi@yahoo.com \\ 2 "Gh. Mihoc - C. Iacob" Institute of Mathematical Statistics and Applied Mathematics, \\ Calea 13 Septembrie 13, RO-050711 Bucharest, Romania \\ Phone/Fax number+40-21-318.. /+40-21-318.24.33, e-mail: alexandru.dumitrache@ima.ro \\ ${ }^{3}$ INCAS - National Institute for Aerospace Research "Elie Carafoli", \\ B-dul Iuliu Maniu 220, Bucharest 061126, Romania \\ Phone/Fax number +40-21-434.00.83/+40-21-434.00.82, e-mail: cismilianu.alexandru@incas.ro
}

\begin{abstract}
A different approach for enhancing the performance of a Vertical Axis Wind Turbines (VAWT) for the use in the urban or rural environment and remote isolated residential areas is developed in this study. Recently the small VAWT became more attractive due to the major advantages of this type of turbines in comparison to the horizontal axis wind turbines. By adding a second set of blades ( $3 \times 2=6$ blades) that following the rules of biplane airplanes we aim to enhance the overall performance of the VAWT. The model has been made to operate at a maximum power in the range of the TSR between 2 to 2.5 . The performances of the VAWT were investigated numerically and experimentally to justify the new proposed design.
\end{abstract}

\section{Key words}

VAWT, Biplane configuration, CFD, Experimental analysis.

\section{Introduction}

Recently, for urban users, the wind turbine with vertical axis (VAWT) has become more attractive due to its benefits in exploitation, the power range usually covering the domain ranging between $2 \mathrm{~kW}$ and $20 \mathrm{~kW}$. As compared to the widely used Horizontal Axis Wind Turbines (HAWTs), VAWTs have many advantages. VAWTs operate with wind blowing from any direction (thus simplifying the wind turbine system), they are designed for low wind speed, and operate at low/medium RPM, have lower vibration and small noise levels and have lower manufacturing and maintenance costs $[1,2]$. Unfortunately, VAWTs have many complicated aerodynamic issues, of which the dynamic stall is an inherent phenomenon which appears at low values of the tip speed ratio (TSR < 4) and has a significant impact on vibration, noise, and power output of the VAWTs. For tip speed ratio $\mathrm{TSR}=1 . .2$ the (theoretical) angle of attack has values over the critical (static) value of the airfoil, forcing the turbine to operate at deep dynamic stall. Hence, to achieve better performance at this operation level (close to the operation level in urban areas) suitable design solutions are necessary. Some of these solutions are based on either passive flow control to ensure increased carrying at high incidences (without a significant increase of the drag) or a biplane configuration.

\section{Urban area placement of a VAWT}

It is well-known that typically the wind flows by the minimal drag path through or over obstacles such as buidings, trees, etc. The borders of these obstacles, i.e., the straight edges of buidings, lead to increased wind speeds of up to 2-3 times the wind speed value of an unperturbed flow. Using the ANSYS Fluent software to analyse the wind flow we have evaluated the effect of the presence of a building within the flow field on three types of buildings (Figure 1). Due to computation limitations we have only simulated the $2 \mathrm{D}$ case. First we have remark that the deviation from the direction of the wind starts long before reaching the obstacle and continues long after leaving it behind. Secondly, we have remark that the flow spectrum depends on the size of the building and the shape of its edges (straight or rounded). Over the building edge the air flows with a certain deviation. Below this particular flow line the wind flow becomes turbulent with high variations of its horizontal speed. The base or the pedestal of the turbine can be built using this flow line as a reference, such that the entire rotor lies in the high speed flow. 


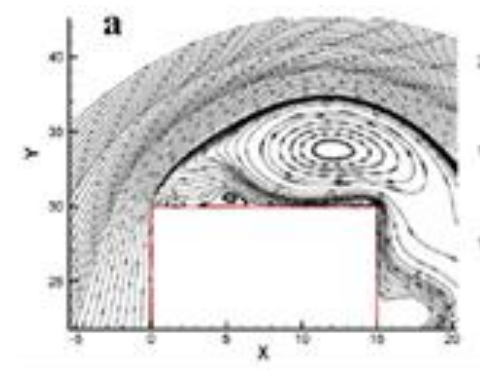

a)

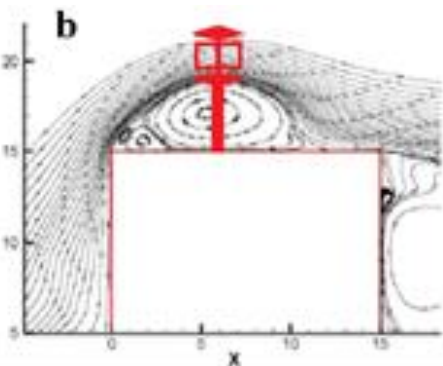

b)

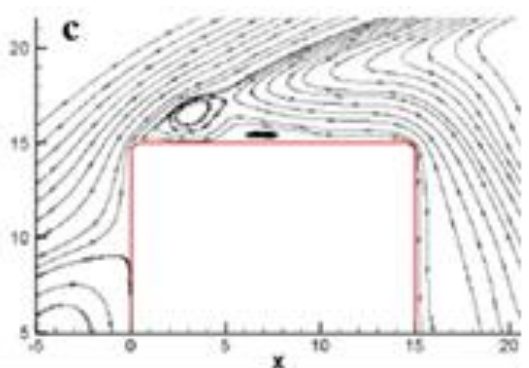

c)

Fig. 1. Various shapes of building: (a) - building profile $30 \mathrm{~m} \times 15 \mathrm{~m}(\mathrm{H} \times 1)$, (b) - building profile $15 \mathrm{~m} \times 15 \mathrm{~m}$, (c) - building profile $15 \mathrm{~m} \times 15 \mathrm{~m}$ with rounded edges $(\mathrm{r}=0.5 \mathrm{~m})$.

The height of the tower of the turbine estimated for the three buildings, for three different locations on the roof with respect to the edge of the building such that the aforementioned criteria are met are presented in Table 1.

Table 1. Tower height for the analysed shapes

\begin{tabular}{|c|c|c|c|}
\hline $\begin{array}{c}\text { Roof } \\
\text { location }\end{array}$ & Profile $-a$ & Profile $-b$ & Profile $-c$ \\
\hline $2.5 \mathrm{~m}$ & $4.0 \mathrm{~m}$ & $3.5 \mathrm{~m}$ & $2.5 \mathrm{~m}$ \\
\hline $5.0 \mathrm{~m}$ & $3.6 \mathrm{~m}$ & $4.1 \mathrm{~m}$ & $4.5 \mathrm{~m}$ \\
\hline $7.5 \mathrm{~m}$ & $7.5 \mathrm{~m}$ & $4.5 \mathrm{~m}$ & $5.5 \mathrm{~m}$ \\
\hline
\end{tabular}

\section{The biplane solution}

The aerodynamic solution for biplane aircraft ensures aerodynamic characteristics at least equivalent to those of a single plane configuration of identical lift area (under the assumption of ideal fluid). In real life, due to the viscous effects, the characteristics yielded by the biplane configuration are equivalent to those of a single plane configuration with an area of 20-40\% larger and identical to the tale of the biplane [3-7]. Figure 2 shows the parameters that define the biplane configuration.

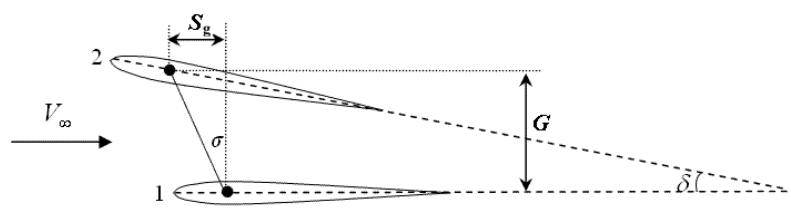

Fig. 2. The geometric elements which the aerodynamic characteristics depend on , i.e., stagger $-S_{\mathrm{g}}$, gap $-\mathrm{G}$, and angle - $\delta$. The positive direction is the one presented in the figure, using the lower wing (lower plane) as reference.

The biplane theory has been developed by Prandtl and Munk. The results of both theories are different from a quantitative perspective, based on the assumptions made, repectively. In the general theory of the biplane, Munk [3] mathematically developes, based on experiment, a formula that describes the lift increase,

$$
\Delta C_{L}= \pm 2 C_{L} \frac{S}{b^{2}}\left(\frac{1}{k^{2}}-\frac{1}{2}\right) \frac{b}{R} \frac{S g}{b},
$$

where $S$ is the total area; $S_{g}$ is the stagger; $b$ is the span; $k$ is the span factor for a single plane equivalent and $R$ is the distance used to compute the air flow deflection behind the configuration. The term

$$
\Delta C_{L}=\left(1 / k^{2}-0.5\right) \cdot b / R
$$

is a function of the ratio between the gap and the span $(G / b)$ called the Munk factor. The influence of the gap increase at the same span: the Munk factor decreases and the unitary lift coefficient $C_{L}$ decreases, too (Figure 3).

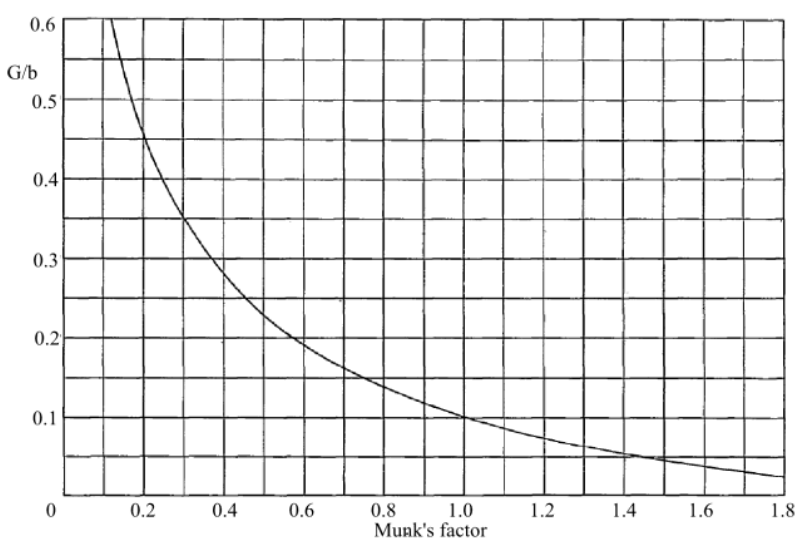

Fig. 3 The Munk factor as a function of the ratio between the gap and the span [3].

The induced drag is calculated using Munk's formula

$$
C_{D, \text { ind }}=C_{L}^{2} S /\left(\pi b^{2} k^{2}\right)
$$

where the variables have the same meaning from the drag coefficient formula. Rough computations yield $k=1$ for all monoplanes and $k \cong 1.1$ for the biplane configuration. This formulae indicate that the gap and the stagger have a significant influences on yielding an aditional lift force.

Experiments in the wind tunnel have been performed on a biplane configuration consisting of two thin airfoils at two Reynolds number of 60,000 and 120,000, respectively [4] (Figure 4). The presented plots emphasize two significant aspects: (1) As expected the biplane possesess lift characteristics which are better than those of an equivalent isolated wing, expecially at high incidences angle; (2) At the same gap, the positive stagger configuration (the upper blade in front of the lower blade - red color) exhibits a $20 \%$ enhancement in the aerodynamics with respect to the negative stagger configuration (blue color) (Figure 4a).

In the works [4-7] the influences of several parameters on the lift coefficient of the biplane have been analysed in detail. Assuming a perfect fluid, using a vortex lattice 
method-based software (Figure 5), extensive research has been done for angles of 5 degrees (Figure 6).

\section{New VAWT and numerical simulation}

The baseline turbine is a Darrieus, H-type with the solidity 0.48 . Keeping a constant radius, add to interior a row of

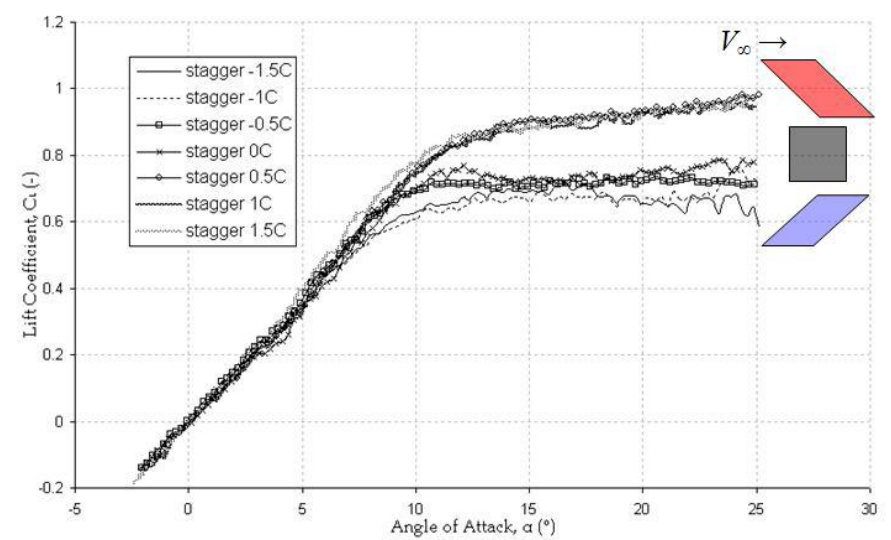

a. three blades with similar airfoil and chord, but staggered with respect to baseline blades with $0.5 \mathrm{c}$ in a tangential direction and staggered across with $0.625 \mathrm{c}$. The numerical investigations on 2D model (experiments are currently underway) led to a higher power coefficient, as expected, compared to classic H-type configuration (Figure 7 and 8).

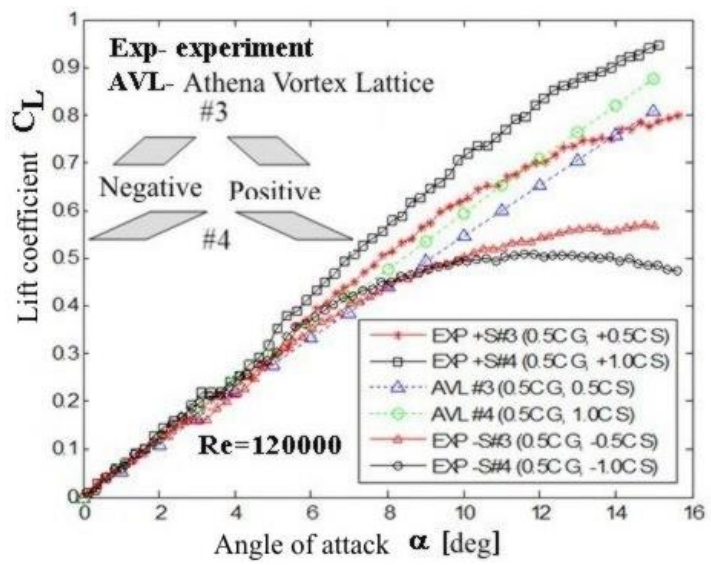

b.

Fig. 4 Experimental results for lift coefficient at $\operatorname{Re}=60,000$ (a.) and $\operatorname{Re}=120,000$ (b.) [4]

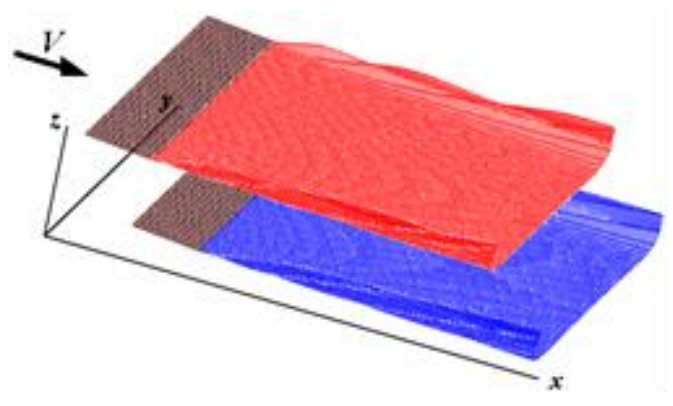

Fig. 5. Vortex lattice method applied to biplane configuration

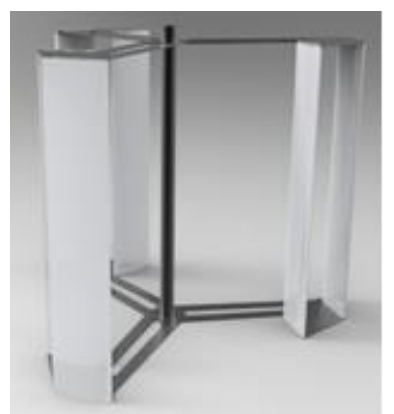

Fig. 7. A 3D view of the biplane type configuration

\section{Passive control methods for VAWTs}

To identify which system of passive control is the most efficient on a typical vertical axis wind turbine, for urban environment, we perform a series of numerical simulations using three systems of passive control: turbulence promoter, thin channel and step. Also, we perform a numerical investigation for biplane configuration. For the present study, we have used Ansys

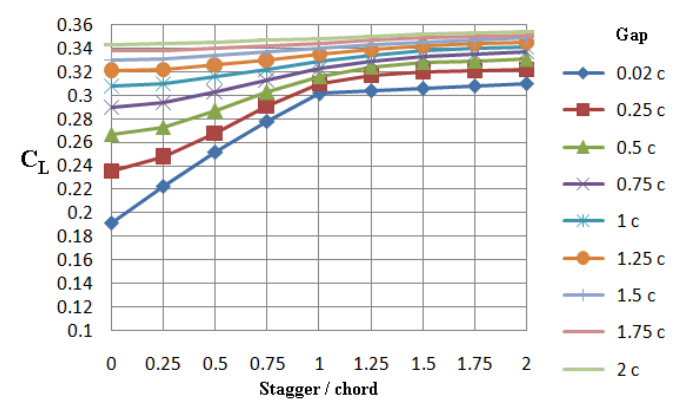

Fig. 6. Influence of gap and stagger on the lift coefficient at incidence angle of $5 \mathrm{deg}$.

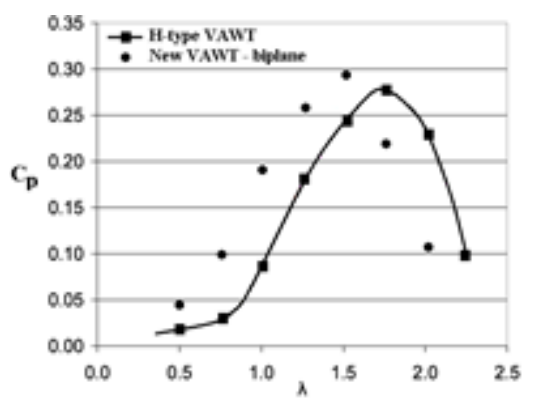

Fig. 8. Power coefficient for the baseline configuration $(\mathrm{H}-$ type) and the proposed configuration (new VAWT - biplane).

Fluent 13 with Reynolds averaged Navier-Stokes (RANS) model and completed with the turbulence model $k-\omega S S T$.

To perform these investigations we have constructed a computational 2D model of the vertical axis wind turbine with 3 blades (airfoil NACA 0018) and 0.48 solidity which is placed in a uniform flow with $15 \mathrm{~m} / \mathrm{s}$ velocity and medium turbulence ( $5 \%$ ), the operating regime 

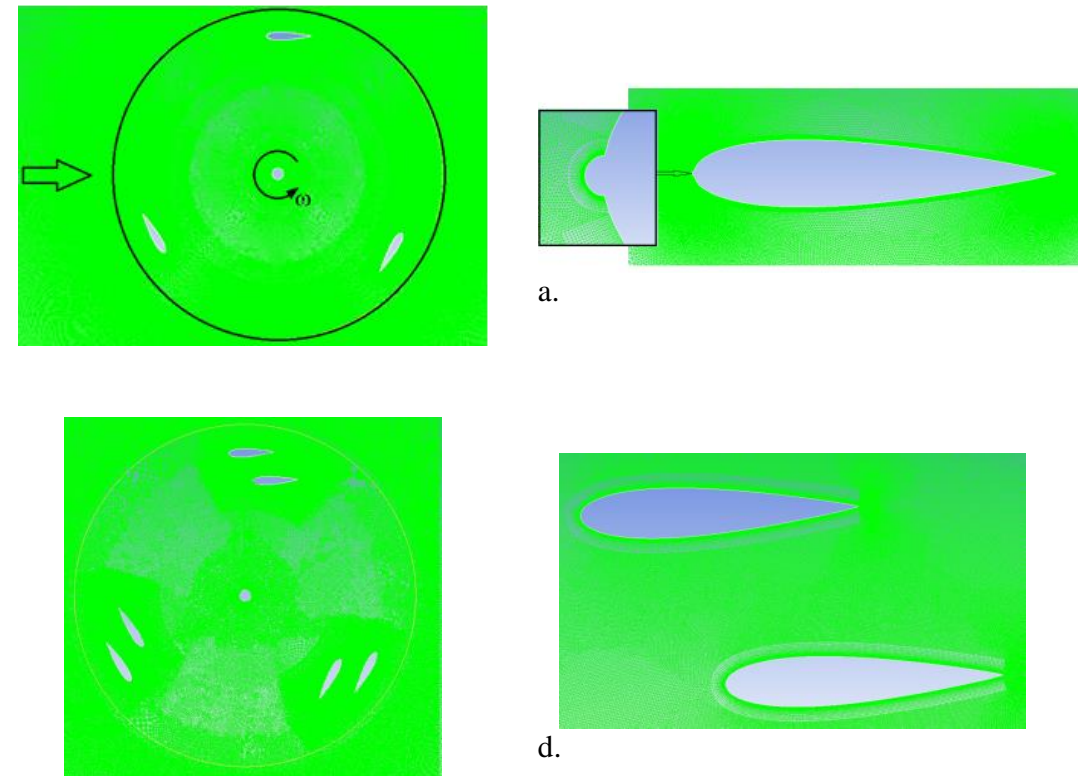

d.

Fig. 9 Computational domain for: a.) Turbulence promoter, b.) Thin Channel, c.) Step, d.) Biplane

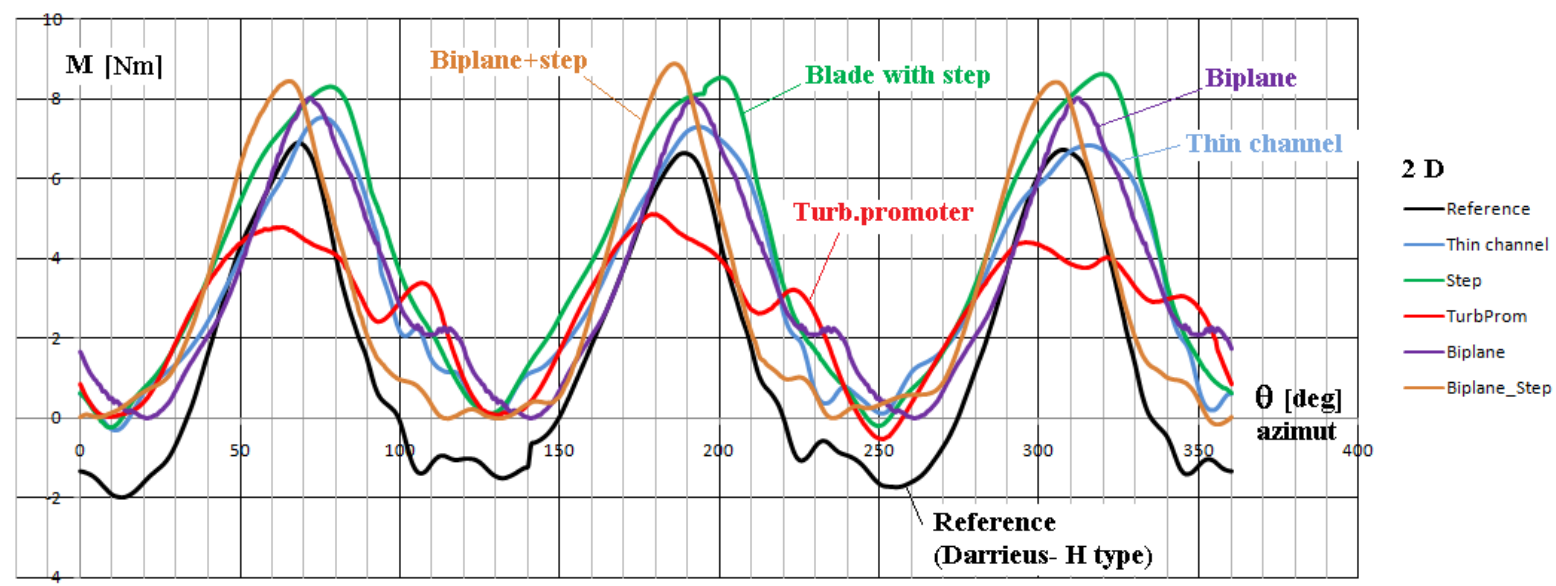

Figure 10. Variation of the aerodynamic momentum of the rotor with respect to the rotation axis ,TSR=1 (for a single rotation)
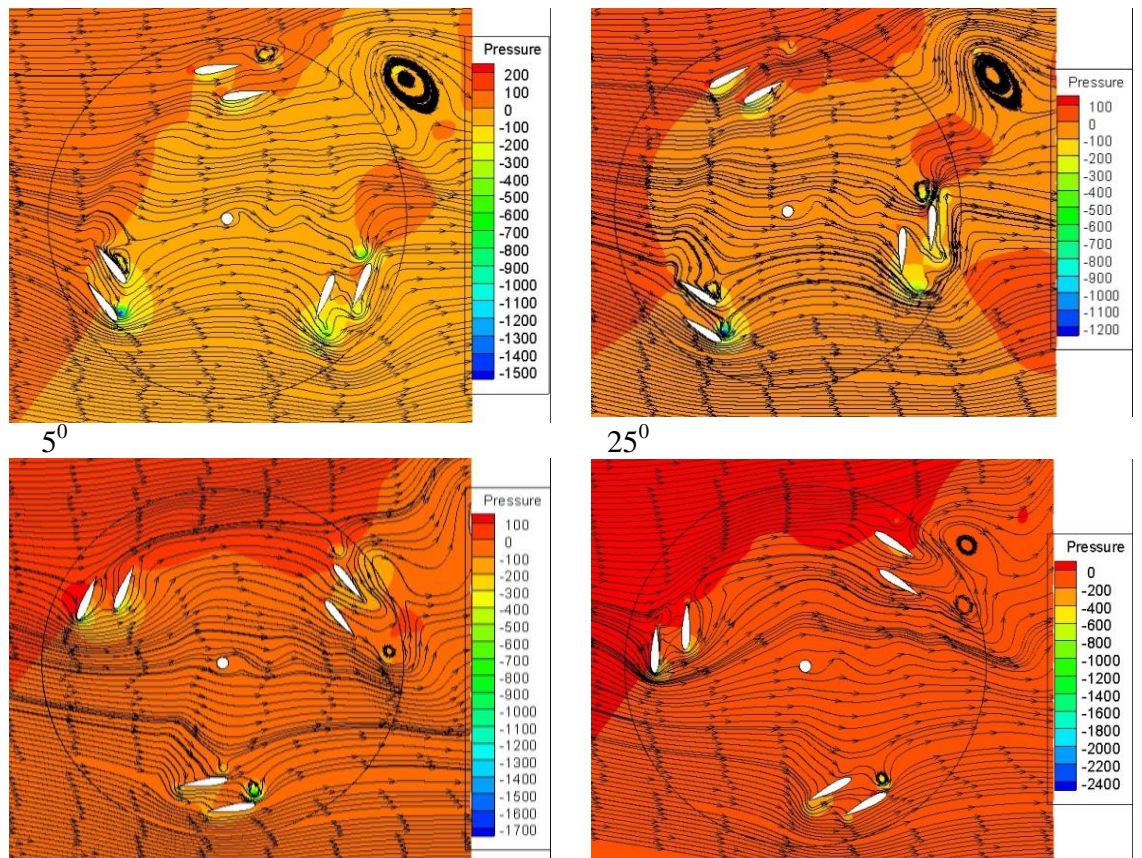

$65^{0}$
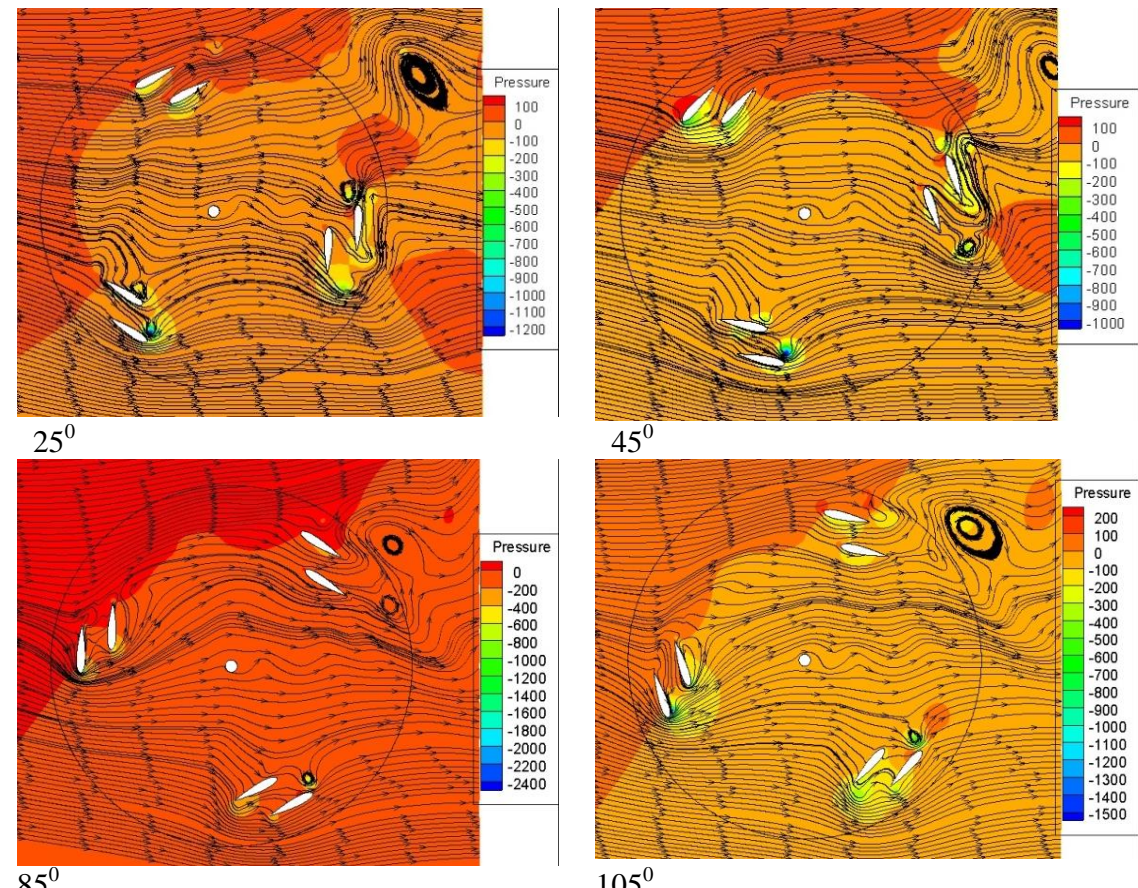

$105^{0}$

Figure 11. Stream lines pattern for the flow around biplane configuration. 
Table 2. The power characteristics for various configurations of VAWT

\begin{tabular}{|c|c|c|c|c|c|}
\hline & $\begin{array}{c}\text { Baseline } \\
\text { configuration }\end{array}$ & $\begin{array}{c}\text { Turbulence } \\
\text { generator }\end{array}$ & Thin jet & Biplane & $\begin{array}{c}\text { Biplane+ } \\
\text { Step }\end{array}$ \\
\hline $\mathrm{M}_{\mathrm{z}}[\mathrm{N} \mathrm{m}]$ & $\mathbf{1 . 5 0 4}$ & $\mathbf{2 . 6 8 6}$ & 3.319 & $\mathbf{3 . 3 1 4}$ & 2.964 \\
\hline $\mathrm{Cp}$ & $\mathbf{0 . 0 8 7}$ & $\mathbf{0 . 1 5 6}$ & $\mathbf{0 . 1 9 3}$ & $\mathbf{0 . 1 9 5}$ & $\mathbf{0 . 1 7 5}$ \\
\hline $\mathrm{C}_{\mathrm{p}, \mathrm{i}} / \mathrm{C}_{\mathrm{p}, 1}$ & $\mathbf{1}$ & $\mathbf{1 . 7 8 6}$ & 2.207 & $\mathbf{2 . 2 3}$ & 2 \\
\hline
\end{tabular}

corresponding to a TSR (tip speed ratio) $=1$. The biplane configuration has size of $0.5 \mathrm{c}$ for the stagger and $0.625 \mathrm{c}$ for the gap.

The computational domain contains two zones: internal zone that contains the rotor which executes a rigid motion with constant angular velocity $60 \mathrm{rad} / \mathrm{sec}$ and fixed external zone (Figure 9). Discretization was made in order to respect all the simulation requirements, the number of nodes is around $6 \times 10^{5}$, and the grid contains both quadrilateral and triangular elements. The time step was chosen on physical aspects and is of the order $\Delta t \cong 10^{-4} \mathrm{sec}$.

Figure 10 shows the variation of the aerodynamic momentum with respect to the rotation axis, at $\mathrm{TSR}=1$ (tip speed ratio $=1$ ), for the reference VAWT and the five passive flow control solutions (biplane among them).

Figure 11 shows the stream lines and the isopressure lines $\left(\Delta p=p-p_{\text {atm }}\right.$ ) for the biplane configuration (motion is periodical, hence only a few snapshots of the flow field are presented). The vortex structure formed by the biplane configuration are illustrated.

Computing the average momentum for a single rotation

$$
\bar{M}_{z}=\frac{1}{T} \int_{0}^{T=2 \pi} M_{z}(\theta) d \theta
$$

and the power coefficient

$$
C_{p}=\frac{\bar{M} \omega}{\rho U^{3} 2 R H / 2}
$$

for $\omega=60 \mathrm{rad} / \mathrm{s}, H=1 \mathrm{~m}, R=0.25 \mathrm{~m}, \rho=1.225 \mathrm{~kg} / \mathrm{m}^{3}$, we get the following synthetized in the Table 2 . It is noticed that for the biplane configuration are obtained higher power coefficient versus monoplane configuration (baseline).

\section{Conclusion}

In this paper we have presented a new model of vertical axis wind turbine (VAWT) using a blade system with a biplane configuration on each arm. Under certain conditions, the biplane configuration yields an increased lift and, depending on the relative position of the blades that form the biplane, yields a high $C_{L} / C_{D}$ ratio, compared to the equivalent monoplane configuration. The preliminary results show that in urban areas, the biplane solution is superior to the classic solution, i.e., of $\mathrm{H}-$ Darrieus type. Additional research of the passive control systems, indicate that they have a positive influence on the VAWT, at TSR of 2 to 3 specific to urban areas. Note that VAWTs can be used efficiently if they use the wind potential augmented by the eaves of the buildings.
Close coupled nonplanar wings can improve $C_{L \max }$ by interferring in a manner similar to slotted flaps (especially at low Reynolds number).

For future work we will continue the numerical and the experimental studies of the proposed configuration, aiming at designing a VAWT with high performances.

\section{Acknowledgement}

This work was realized through the Partnership programme in priority domains - PN II, developed with support from ANCS CNDI - UEFISCDI, project no. PNII-PT-PCCA-2011-3.2-167.

\section{References}

[1] "HAWT versus VAWT: Small VAWTs find a clear niche" Refocus 4, 2003, pp. 44-46.

[2]. I. Nila, R. Bogateanu and M. Stere, "Small power wind turbine (Type Darrieus)", INCAS BULLETIN, 4, 2012, pp. 135-142.

[3] M. M. Munk, "General Biplane Theory", NACA Technical Report TR-151, 1929.

[4] H. Kang, N. Genco and A. Altman, "Gap and Stagger Effects on Biplanes with End Plates Part I." 47th AIAA Aerospace Sciences Meeting Including The New Horizons Forum and Aerospace Exposition 5 - 8 January 2009, Orlando, Florida.

[5] Corso di Laurea Magistrale in Ingeneria Aeronautica, "The Tandem Wing: Theory, Experiments, and Practical Realizations" Relatore: Prof. Lorenzo Trainelli, Candidato: Angelo Minardo, matr. 725266, Anno accademico 2013 / 2014.

[6] A. Maqsood and G. T. Hiong, "Parametric Studies and Performance Analysis of a Biplane Micro Air" Int. J. of Aeronautical \& Space Sci. 14, 2013, pp. 229-236.

[7] A. Quental, M. D. Singh, J. Minz, Task 1: "Study of Biplane Aerodynamics", Project: Sea-Biplane, ITSP 2012: Weekly Report 1 (6 to 11-May-2012). 